\title{
Treatment of Gastrointestinal Bleeding in a Probable Case of Cerebroretinal Microangiopathy with Calcifications and Cysts
}

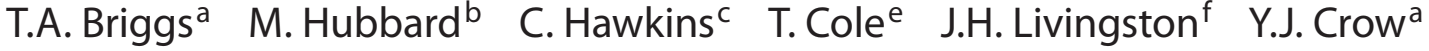 \\ A. Pigott ${ }^{d}$ \\ ${ }^{a}$ Genetic Medicine, University of Manchester, Manchester Academic Health Science Centre, Manchester, \\ Departments of ${ }^{b}$ Paediatrics, ${ }^{c}$ Neurology, and ${ }^{d}$ Pediatric Gastroenterology, University Hospital of North \\ Staffordshire, Stoke-on-Trent, 'West Midlands Regional Genetics Service, Birmingham Women's Hospital \\ Healthcare NHS Trust, Birmingham, and f Department of Paediatric Neurology, Leeds General Infirmary, Leeds, UK
}

\section{Key Words}

Cerebroretinal microangiopathy $\cdot$ Coats plus $\cdot$ CRMCC •

Cysts $\cdot$ Gastrointestinal bleeding $\cdot$ GAVE $\cdot$ Intracranial calcification

\begin{abstract}
Cerebroretinal microangiopathy with calcifications and cysts (CRMCC) is a highly pleiotropic disorder, particularly affecting the eye, brain, bone, and gut. The potential catastrophic sequelae of the associated gastrointestinal phenotype, variably characterised by both chronic bleeding and liver failure, is becoming increasingly apparent. Here we report a probable case of CRMCC with pre- and postnatal growth restriction, bilateral exudative retinopathy, a pathognomonic pattern of intracranial calcification, white matter disease, osteopenia with a tendency to fractures, and chronic gastrointestinal bleeding secondary to abnormal dilated vasculature. The gastrointestinal endoscopic findings were characteristic of gastric antral vascular ectasia (GAVE). Treatment with a combination of oral oestrogen and progesterone ameliorated the gastrointestinal blood loss such that monthly blood transfusions could be stopped. The benefit of this relatively benign therapy in managing the potentially life-limiting consequences of an abnormal gastrointestinal vasculature in CRMCC is of great interest.
\end{abstract}

Copyright $\odot 2010$ S. Karger AG, Basel

\section{KARGER}

Fax +4161306 1234

E-Mail karger@karger.ch

www.karger.com (c) 2010 S. Karger AG, Basel

$1661-8769 / 10 / 0014-0159 \$ 26.00 / 0$

Accessible online at:

www.karger.com/msy
Cerebroretinal microangiopathy with calcifications and cysts (CRMCC) is a multi-system disease showing variable involvement of the eyes, brain, bone, and gut. We recently described 9 cases highlighting the core clinical features of this condition which are: pre- and postnatal growth retardation, bilateral retinal telangiectasia and exudates (Coats disease); intracranial calcification, a leukoencephalopathy sometimes associated with parenchymal cysts; osteopenia with a tendency to fracture, bone marrow suppression and gastrointestinal bleeding with cirrhosis. Less frequently, patients demonstrate sparse, grey hair, dystrophic nails and café au lait patches [Briggs et al., 2007]. It is becoming increasingly apparent that gastrointestinal involvement with bleeding and liver failure represent major causes of morbidity and mortality in this disorder [Linnankivi et al., 2006; Briggs et al., 2007].

Considering reports of affected sibling pairs, equally severely affected males and females, and the presence of consanguinity in some families, CRMCC is likely inherited as an autosomal recessive trait. However, the precise genetic basis of this multisystem microangiopathy remains uncertain at this time. We have derived data to suggest that CRMCC is not allelic with other known monogenic neurovascular microangiopathies including CADASIL (NOTCH3), COL4A1-associated disease and retinal vasculopathy with cerebral leukodystrophy (TREX1). 

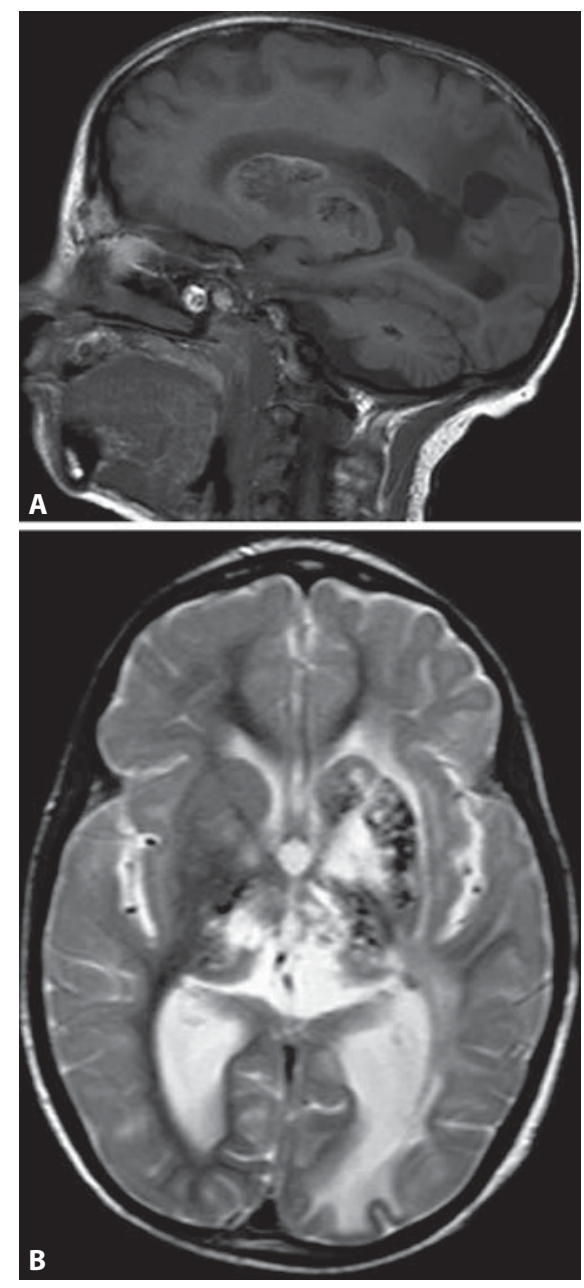

Fig. 1. Brain MRI performed at age 27 years. A Sagittal T1-weighted scan shows extensive involvement of the basal ganglia consistent with calcification, and a cystic lesion of the left parietal lobe. B T2-weighted axial image demonstrating asymmetric white matter high signal in the left parieto-occipital region, and extensive, asymmetric calcification of the basal ganglia.

Here we report a probable case of CRMCC where chronic gastrointestinal bleeding previously necessitating recurrent transfusion was ameliorated with the use of hormonal therapy.

\section{Case History}

This female was born at 37 weeks of gestation with a birth weight of $1.3 \mathrm{~kg}(<0.4$ th centile). She had been small for dates throughout the pregnancy. At birth she was noted to have absent thumbs, a dislocated right radial head and dislocatable hips. Investigations at this time included a negative torch screen, normal female karyotype and exclusion of Fanconi's anaemia.
She was the fourth pregnancy of nonconsanguineous healthy parents. The first ended in miscarriage, the second in a stillborn male at 34 weeks who was reported to have a normal appearance and the third a live born healthy female.

In early infancy the patient required multiple orthopaedic procedures, including a left hip osteotomy and correction of genu varum and equinovarus deformities. Despite this she walked independently at 18 months. Her growth remained below the 3rd centile for all parameters.

At age 2 years, she was noted to have hearing impairment reportedly secondary to an ear canal deformity. At 3 years of age, she presented with a convergent squint and her visual acuity was found to be $3 / 60$ bilaterally. On fundoscopy, marked sub-exudation was seen at the posterior poles of both eyes. A clinical diagnosis of bilateral Coats disease was made.

At age 8 years, she fractured her right femur following minimal trauma. This was to be the first of several femoral and tibial fractures in which healing was extremely problematic. The severity of her skeletal symptoms was such that she was investigated for osteogenesis imperfecta. However, $\mathrm{N}$ terminal procollagen peptide, bone alkaline phosphatase and osteocalcin were within normal limits. At age 25 years, despite attempts at bone fixation and grafting, a right transarticular knee amputation for chronic nonunion of the tibia and osteomyelitis was required.

In view of a 9-year history of absence and myoclonic seizures, microcephaly and mild developmental delay, a CT scan was performed at 14 years of age. This showed dilated ventricles together with marked calcification of the choroid plexus and coarse calcification of the basal ganglia and thalami bilaterally. Ischaemic changes were reported in the left parieto-occipital region. More recently, at the age of 27 years a brain MRI demonstrated left cerebral hemisphere leukodystrophy and widespread calcification (fig. 1).

On examination at 16 years of age, it was noted that she had very fine hair, which was particularly sparse frontally, and hypoplastic toe nails and teeth. She was $94 \mathrm{~cm}$ tall $(<-10$ SD below mean) with a small head (measurement unrecorded), bilaterally blind secondary to cataracts and glaucoma, and partially deaf.

In her teens she developed a chronic iron deficient anaemia (haemoglobin $<7 \mathrm{~g} / \mathrm{dl}$ ), the cause of which was initially undefined. At 20 years of age, she presented with haematemesis. An endoscopy was reported as showing severe haemorrhagic antral gastritis with pre-pyloric ulcers and erosions. Histology of the gastric antral mucosa revealed oedema of the lamina propria, a mild increase in smooth muscle fibres within the lamina propria and dilated blood vessels.

Her gastrointestinal (GI) problems persisted with recurrent haematemesis. However, liver function tests and liver ultrasound including dopplers of portal flow were all normal, making cirrhosis/portal hypertension unlikely. A further endoscopy was performed following a severe episode of haematemesis at 22 years of age. This revealed abnormal vasculature most prominent in the pyloric canal (fig. 2). The appearance was considered characteristic of 'watermelon stomach' or gastric antral vascular ectasia (GAVE). The histology was also consistent with GAVE. Argon laser therapy was commenced to good effect. However, a year later during her 6th procedure she suffered an iatrogenic stomach perforation.

Haematemesis continued and by the age of 25 years monthly blood transfusion was required to control her anaemia. She was 
commenced on a trial of hormone therapy, norethisterone $1 \mathrm{mg}$ and ethinyloestriadiol $20 \mu \mathrm{g}$, once daily for 63 days followed by a 7 -day break (the infrequent breaks were prescribed to reduce the number of withdrawal bleeds for patient comfort). This had a remarkable effect on her gastrointestinal bleeding. At the age of 27 years, her haemoglobin level was $11.5 \mathrm{~g} / \mathrm{dl}$, and she had not needed a transfusion since treatment was commenced 2 years previously.

Beginning at the age of 28 years, she developed increasing dyspnoea. CT scan showed features consistent with multilobar interstitial fibrosis with traction bronchiectasis. Lung biopsy was not performed. She died of respiratory failure 9 months after the onset of her respiratory symptoms. Post-mortem studies were not undertaken.

\section{Discussion}

The clinical diagnosis of CMRCC seems highly likely in this case in view of the pre- and postnatal growth restriction, bilateral exudative retinopathy, characteristic pattern of intracranial calcification and leukoencephalopathy, and significant gastrointestinal bleeding. The osteopenia and tendency to fractures is also in keeping with the diagnosis. We have previously reported a typical case of CRMCC in whom osteopenia was so severe that a diagnosis of osteogenesis imperfecta was initially considered [Briggs et al., 2007]. However, we note that the degree of short stature, absence of the thumbs and deafness seen in our case have not previously been reported in CRMCC. For this reason we cannot be absolutely certain of the diagnosis and await a genetic test to clarify this matter.

Recurrent, severe GI haemorrhage as seen in this case is increasingly recognised in CRMCC. Linnankivi et al. [2006] reported 6 patients with CRMCC presenting with transfusion dependent anaemia secondary to occult intestinal bleeding. Three patients described in our previous publications have subsequently experienced life limiting GI bleeding (patients 1, 2 and 3) [Crow et al., 2003]. In addition, 2 patients reported by Linnakivi et al. [2006] and 1 of our patients (patient 1) developed hepatic insufficiency and varices [Crow et al., 2003].

Pathological examination of eye and brain biopsies in CRMCC suggests that the underlying basis of the CNS disease is an obliterative microangiopathy with vessels showing thickened, sclerotic and calcified walls [Briggs et al., 2007]. In patients experiencing gastrointestinal bleeding and liver involvement, biopsies have demonstrated portal fibrosis with abnormally large vascular channels and telangiectasia in the small bowel with thick-walled, dilated blood vessels [Linnankivi et al., 2006; Briggs et al., 2007].

Bleeding in CRMCC

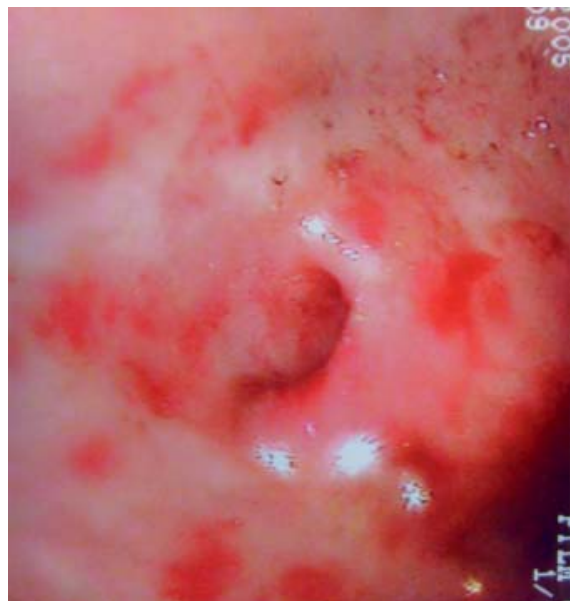

Fig. 2. Endoscopy preformed at 22 years of age. Areas of erythema represent vascular ectasia. Within the gastric antrum (image unavailable) the erythematous lesions extend proximally along the length of the stomach to produce a so-called 'watermelon stomach'.

In the current case, endoscopic features were considered characteristic of GAVE, a diagnosis which prompted the initiation of hormonal therapy. GAVE was first described in 1953 as 'an erosive atrophic gastritis with marked venocapillary ectasia' [Rider et al., 1953]. On endoscopy, the stomach is said to resemble a watermelon, with prominent erythematous stripes radiating from the pylorus to the antrum [Sebastian et al., 2003].

Sebastian et al. [2003] acknowledge that there can be difficulty in differentiating GAVE from portal hypertensive gastropathy concomitant with cirrhosis. Indeed, 30\% of GAVE patients are reported to have associated cirrhosis. In the current case, whilst the histological diagnosis was consistent with GAVE, it was noted that the changes would also fit with portal gastropathy. GAVE most commonly presents as iron deficiency anaemia in elderly females [Gostout et al., 1992], whilst CRMCC patients reported to date have been children or young adults.

Of particular importance in our case was the beneficial effect of hormonal therapy on GI bleeding. The use of oestrogen medication resulted in a dramatic modification of the disease phenotype so that monthly blood transfusions which were at one time essential were not required for over 2 years. In a review of current therapies in GAVE, Sebastian et al. [2003] state that a combination of oestrogen and progesterone successfully controls bleeding, or at least reduces transfusion requirements, in GAVE patients. However, the underlying vascular malformation is not modified and bleeding is likely to recur 
if treatment is withdrawn. Oestrogens exert important regulatory functions on vessel wall components in responses to endothelial inflammation, injury and repair, although the precise mechanisms through which oestrogen regulates endothelial cell function is complex and poorly understood [Cid et al., 2002].

Although the underlying aetiology of her pulmonary disease remains unclear, there are no previous reports of the progesterone/oestrogen preparation with which this patient was treated causing pulmonary fibrosis. We remain suspicious that she suffered a reactive fibrosis secondary to pulmonary microbleeds, reflective of the systemic microangiopathy underlying CRMCC.
In summary, we report a case of CRMCC with gastrointestinal bleeding secondary to abnormal dilated GI vasculature responding to hormonal therapy. The positive impact of such therapy on this life limiting feature of CRMCC is of potential importance. However, confirmation of efficacy will require evidence of benefit in other cases.

\section{Acknowledgements}

We would like to thank the family for their involvement in this study. Y.J.C. acknowledges the Manchester NIHR Biomedical Research Centre, and the Birth Defects Foundation Newlife.
References
Briggs TA, Abdel-Salam GM, Balicki M, Baxter P, Bertini E, et al: Cerebroretinal microangiopathy with calcifications and cysts (CRMCC). Am J Med Genet A 146A:182-190 (2007).

-Cid MC, Schnaper HW, Kleinman HK: Estrogens and the vascular endothelium. Ann NY Acad Sci 966:143-157 (2002).

-Crow YJ, McMenamin J, Haenggeli CA, Hadley DM, Tirupathi E, et al: Coats' plus: a progressive familial syndrome of bilateral Coats' disease, characteristic cerebral calcification, leukoencephalopathy, slow pre- and post-natal linear growth and defects of bone marrow and integument. Neuropediatrics 35:10-19 (2003).
Gostout CJ, Viggiano TR, Ahlquist DA, Wang $\mathrm{KK}$, Larson MV, et al: The clinical and endoscopic spectrum of the watermelon stomach. J Clin Gastroenterol 15:256-263 (1992).

Linnankivi T, Valanne L, Paetau A, Alafuzoff I, Hakumaki JM, et al: Cerebroretinal microangiopathy with calcifications and cysts. Neurology 67:1437-1443 (2006).

Rider JA, Klotz AP, Kirsner JB: Gastritis with veno-capillary ectasia as a source of massive gastric hemorrhage. Gastroenterology 24: 118-123 (1953).

- Sebastian S, O’Morain CA, Buckley MJ, Review article: current therapeutic options for gastric antral vascular ectasia. Aliment Pharmacol Ther 18:157-165 (2003).
Briggs/Hubbard/Hawkins/Cole/ Livingston/Crow/Piggott 